\title{
Erratum: Linear analysis of coupled lattices \\ [Phys. Rev. ST Accel. Beams 2, 074001 (1999)]
}

\author{
D. Sagan and D. Rubin
} (Published 23 May 2000)

PACS numbers: 29.20.Dh, 29.27.Bd

It has been called to our attention that two equations in our paper are wrong.

Equation (13) should read

$$
\mathbf{A}=\gamma^{2} \mathbf{M}-\gamma\left(\mathbf{C n}+\mathbf{m C}^{+}\right)+\mathbf{C N C}^{+},
$$

and Eq. (14) should read

$$
\mathbf{B}=\gamma^{2} \mathbf{N}+\gamma\left(\mathbf{n C}+\mathbf{C}^{+} \mathbf{m}\right)+\mathbf{C}^{+} \mathbf{M C} .
$$

These errors do not affect any other part of the paper nor do they affect the conclusions therein. Our thanks to Dr. Stuart Henderson for pointing out the error in the paper. 